\title{
Analysis of Cognitive Radio Spectrum Sensing Techniques over Nakagami-m Fading Channel
}

\author{
Mohammad Shahnawaz Shaikh, P. K. Khare \\ Department of Post Graduate Studies and Research in Physics and Electronics \\ Rani Durgavati Vishwavidyalaya Jabalpur, India
}

\begin{abstract}
As the demand of wireless communication increases exponentially, with the same ratio scarcity of spectrum also originates. To overcome this spectrum scarcity a novel approach, Cognitive Radio (CR) shows development of an opportunistic and promising technology. This paper explores implementation and analysis of the CR spectrum sensing techniques such as Matched filtering, Energy detection and Cyclostationary feature detection on MATLAB platform by simulation. We analyze performance of these techniques over, Nakagami-m fading channel with AWGN channel for both the BPSK and QPSK modulation.

Keywords: Cognitive Radio, Spectrum sensing, Energy detection, Matched filtering, Cyclostationary detection,Nakagami-m fading channel, AWGN channel, BPSK, QPSK
\end{abstract}

\section{INTRODUCTION}

Cognitive radio $(\mathrm{CR})$ is an excellent development to treat the scarcity of spectrum. As the wireless communication is spreading its application exponentially at commercial as well as domestic level, dynamic spectrum allocation play an effective role to reduce the problem of spectrum scarcity [1][10]. In our implementation we show the performance of cognitive radio spectrum sensing techniques like Energy detection, Matched filtering and cyclostationary feature detection over the Nakagami $\mathrm{m}$-fading channel. Individual performance of each sensing techniques analyzed for both BPSK and QPSK modulation. We plot the curve for SNR vs Decision accuracy for each sensing techniques although combine analysis also done for both BPSK and QPSK modulation techniques over Nakagami-m fading channel.

This paper organised as, first section introduced to our work. Matched filtering, Energy detection and Cyclostationary feature detection techniques briefly introduces in the section second, third and fourth respectively with their basic mathematical expressions. Nakagami-m fading channel discussed in the section five. Section six shows the performance of discussed spectrum sensing techniques individually. Finally section seven concluded the inferences drawn from our work.

\section{MATCHED FILTERING}

Matched filtering is an optimum and most promising method of spectrum sensing. It require prior knowledge of primary user signals like operating frequency, modulation type, packet format, bandwidth and pulse shaping etc. Matched filter is a linear filter which perform coherent detection of primary user (PU) signals. In its operation it performs correlation of received primary user signal with the filter impulse response to check the spectrum is busy or idle. although its performance

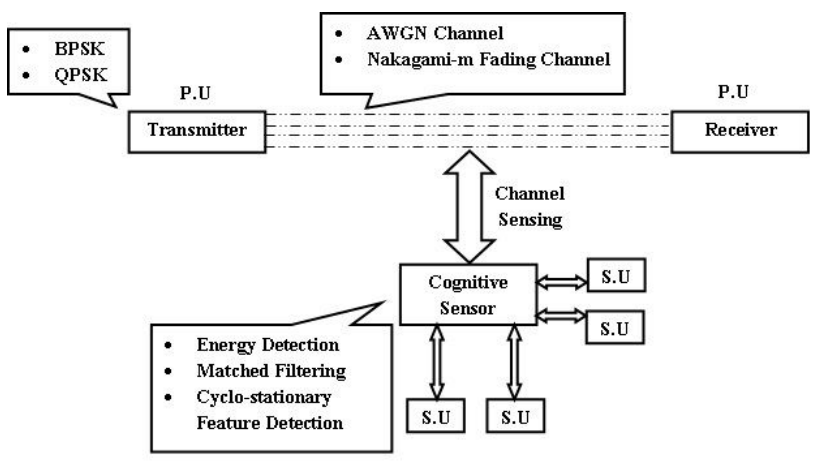

Fig. 1. Cognitive environment over propagation channels

might be week in the absence of suitable desired primary user information. [1] - [4]. As the PU signal passes through the matched filter, it produces a peak in its output to show the presence of PU signal over sensed spectrum. At the same time it increases the signal component and decreases the noise component to maximize SNR [12].

$$
Y[n]=\sum_{k=-\infty}^{+\infty} h[n-k] z[k]
$$

Where $z$ is unknown signal and is convolved with the $h$, the impulse response of matched filter that is matched to the reference signal for maximizing the SNR [1], [2], [3], [9].

\section{ENERGY DETECTION}

Energy detection is very simple and effective spectrum sensing technique. It is most popular and economic due to its simplicity. It is based on non-coherent detection and does not need prior knowledge of structure or signal format of PU signal. The energy detector calculate the signal energy over the desired spectrum and compare it with pre calculated threshold energy level. If the calculated energy level is found to be greater than threshold energy level, it is considered as desired spectrum is busy with PU signals. The desired sspectrum is said to be free or idle when energy detector found energy level over the desired spectrum is less than threshold energy level. Now CR engine can offer this spectrum to Secondary User (SU) [1]-[4],[7], [9], [10], [11], [13] .

$$
S_{N}(x)=\frac{1}{N} \sum_{n=-1}^{N} S(n)^{2}
$$


Here $S_{N}(x)$ is energy calculated by energy detector. The threshold energy level $T h$ calcuated by using decision paprameter $D$ as

$$
D=\frac{P_{a v g}}{P_{m}}
$$

Where $P_{\text {avg }}$ is average power while $P_{m}$ is mean power

$$
\begin{aligned}
T h & =\frac{\max (D)+\min (D)}{2} \\
X(n) & = \begin{cases}N(n) & H_{0} \\
S(n)+N(n) & H_{1}\end{cases}
\end{aligned}
$$

Hypotheis $H_{0}$ shows the absence of PU signal over desired spectrum while $H_{1}$ indicates spectrum is sharing PU information.

\section{CYCLOSTATIONARY FEATURE DETECTION}

Cyclostationary or repeating features of any communication signal are the underlying properties of that signal, which can be used for its detection over the communication channel. Autocorrelation and cyclic spectral density of primary user (PU) signal exhibits periodicity introduced in the signal format. Cyclostationary feature of PU signal characterised by periodicity like, sine wave carriers, pulse trains, repeating sequences, spreading, hoping sequences, or cyclic prefixes. Receiver performs signal detection over communication channel by exploiting the cyclic features of PU signal while this cyclic features of PU are previously stored in the memory of cognitive engine. This spectrum sensing technique does not require frequency or phase synchronization of signal while performs better than energy detection technique in for low signal to noise ratio (SNR). However this technique has higher degree of complexity than other popular spectrum sensing techniques as it makes large calculation [1], [2], [4], [8].

The received signal is given by

$$
X(n)=s(n)+w(n)
$$

The cyclic spectral density (CSD) function of a received signal can be calculated as

$$
S(f, \alpha)=\sum_{\tau=-\infty}^{\infty}\left(R_{Y}^{\alpha}\right)(T) e^{-2 j \pi f T}
$$

Where cyclic autocorrelation function (CAF) given by

$$
\left(R_{Y}^{\alpha}\right)(T)=E\left[y(n+\tau) y(n-\tau) e^{2 j \pi \alpha n}\right]
$$

Here $\alpha$ is the cyclic frequency. CSD function exhibits its maximum values, when fundamental frequency of transmitted signal $x(n)$ is equal to the the cyclic frequency [2], [3].

\section{NAKAGAMI-M FADING CHANNEL}

In the practical wireless communication, multihope and multipath transmission is the most occuring scenarios. Nakagami-m fading channel is the most suitable fading enviroment to summarize such types of propagation path [14]. It is more accurate and better than the Rayleigh and Rician fading channel as it has $m$ diversity path. Probability density function of Nakagami-m fading channel is given by

$$
f\left(P_{r}\right)=\frac{P_{r}{ }^{2 m-1} 2 m^{m}}{(m-1) !{\overline{P_{r}}}^{2 m}} \exp \frac{-m}{{\overline{P_{r}{ }^{2}}}^{2}} P_{r}^{2}
$$

Where $P_{r}$ is the Nakagami distribution envelope and $m$ is shape factor, which is given by

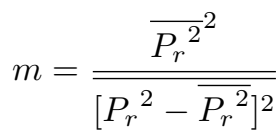

For $m=1$ its performance gets equivalent to Rayleigh fading channel [13], [14] Nakagami distribution comprehend direct components with the reflected and scattered component of original transmitted signal.

\section{RESULTS AND ANALYSIS}

We simulate our work on MATLAB to analyse performance of CR spectrum sensing techniques over the Nakagami-m fading channel and AWGN channel. Analysis done by observing plots, SNR Vs Decision accuracy (Detection probability) for BPSK and QPSK modulation technique. This work done for Matched filtering, Energy detection and Cyclostationary feature detection spectrum sensing technique.

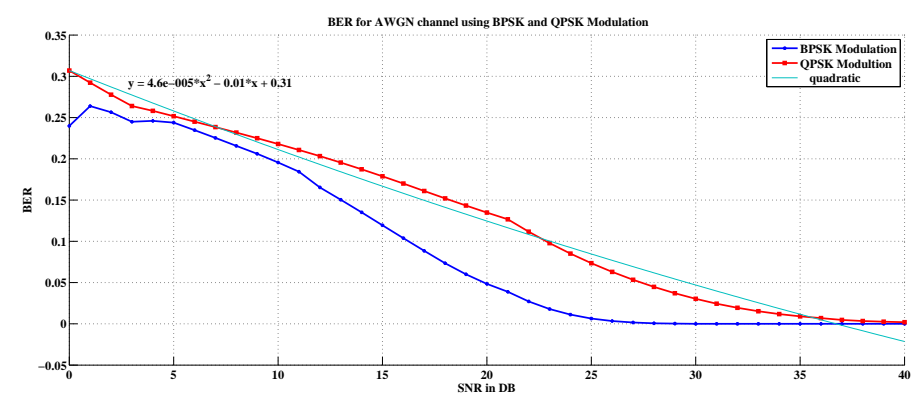

Fig. 2. BER over AWGN channel using BPSK and QPSK modulation

Figure 2 shows the BER over AWGN channel for BPSK and QPSK modulation. BER decrease rapidly as SNR increase for BPSK modulation as compared to QPSK modulation while curve for QPSK modulation showing linearity as compared to curve for BPSK modulation

Figure 3 shows the BER over Nakagami-m fading channel for BPSK and QPSK modulation. BER is almost constant over the whole SNR range for both the BPSK and QPSK modulation. It is imortant to observe that for QPSK modulation BER is very low. It can be considered as errorless transmission. 


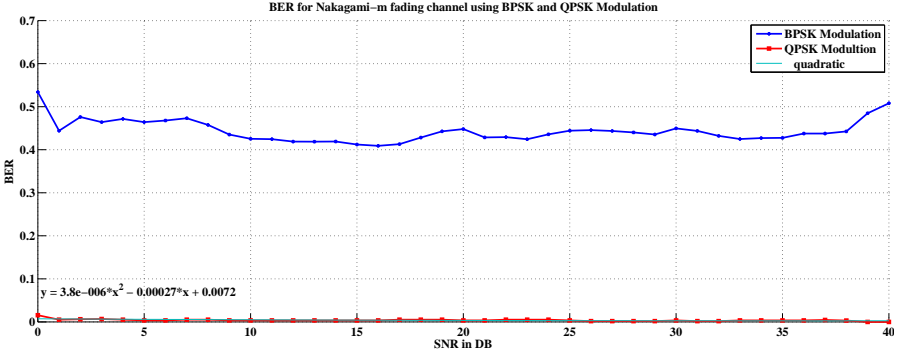

Fig. 3. BER over Nakagami-m fading channel using BPSK and QPSK modulation

\section{A. Matched Filtering}

Performance of Matched filtering technique calculated over AWGN channel and Nakagami-m fading channel for both BPSK and QPSK modulation

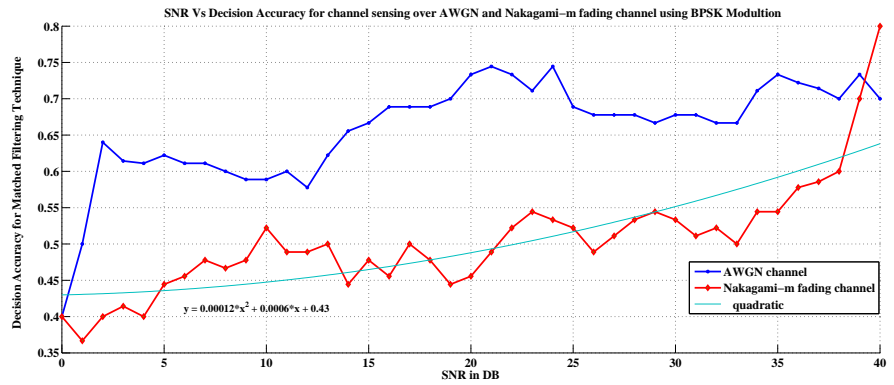

Fig. 4. Decision accuracy of Matched filtering technique over AWGN and Nakagami-m fading channel using BPSK modulation

Figure 4 and figure 5 shows decision accuracy of Matched filtering technique over AWGN channel and Nakagami-m fading channel for BPSK modulation QPSK modualtion respectively. Matched filtering technique offering better performance over AWGN channel rather than Nakagami-m fading channel for BPSK modulation, while for QPSK modulation, Nakagami-m fading channel gives better performance than AWGN channel for low SNR values. It is also observed that for high SNR values performance over both the channel gets equivalent.

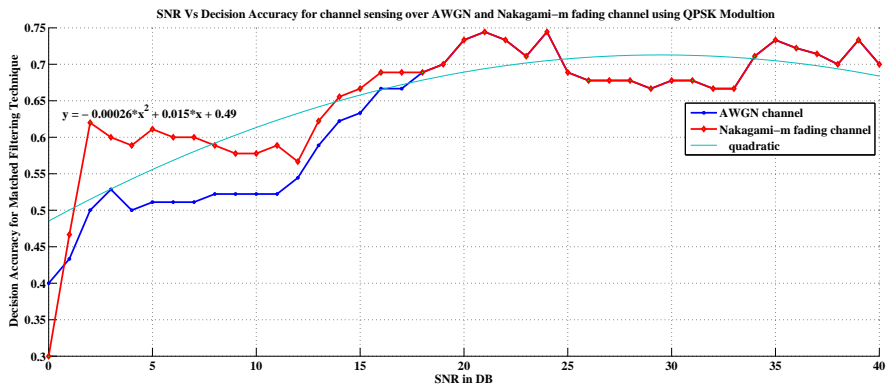

Fig. 5. Decision accuracy of Matched filtering technique over AWGN and Nakagami-m fading channel using QPSK modulation

Figure 6 shows individual performance of Nakagami-m fading channel for both BPSK as well as QPSK modulation.
This channel offer better performance for QPSK modulation rather than BPSK modulation.

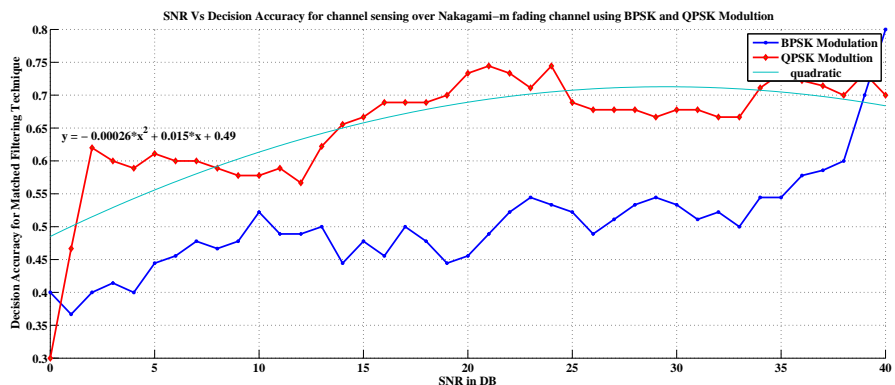

Fig. 6. Decision accuracy of Matched filtering technique over Nakagami-m fading channel using BPSK and QPSK modulation

\section{B. Energy Detection}

Decision accuracy for energy detection technique showing good results over AWGN channel with respect to Nakagami$\mathrm{m}$ fading channel for both the BPSK and QPSK modulation. It can be analyze in figure 7 and 8 . However Nakagami-m fading channel offer better performance for QPSK modulation as shown in figure 9 .

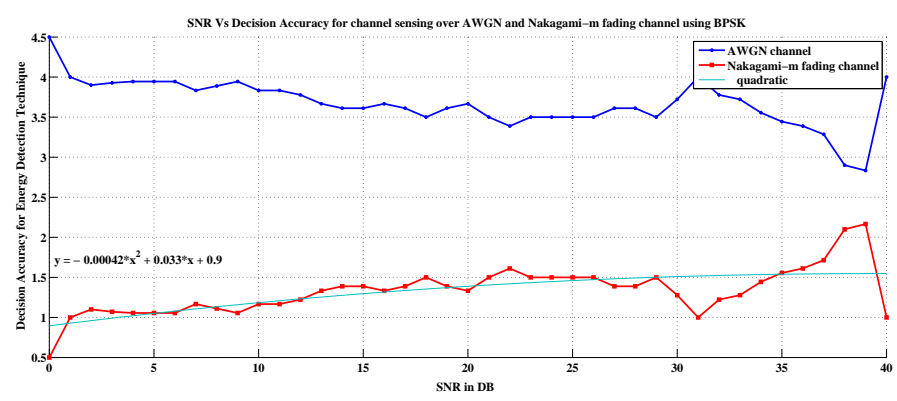

Fig. 7. Decision accuracy of Energy Detection technique over AWGN and Nakagami-m fading channel using BPSK modulation

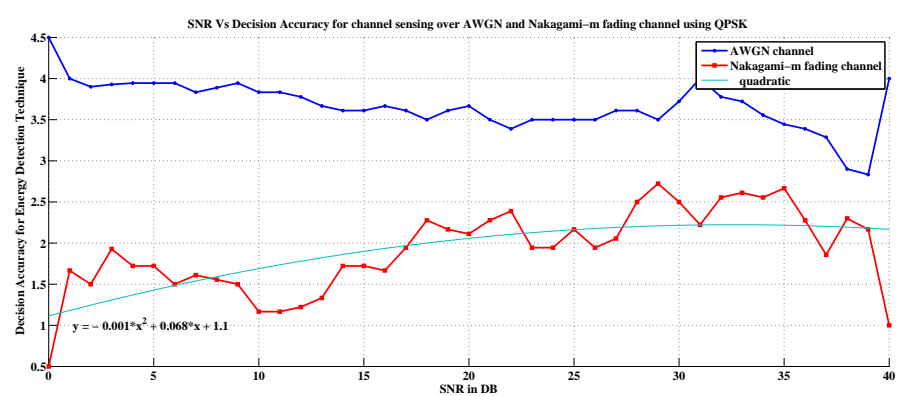

Fig. 8. Decision accuracy of Energy Detection technique over AWGN and Nakagami-m fading channel using QPSK modulation

\section{Cyclostationary feature detection}

This technique is one of the more accurate spectrum sensing technique. Effect of noise is alomst neigligible on its performance. It can easily be seen in the figure 10 and figure 


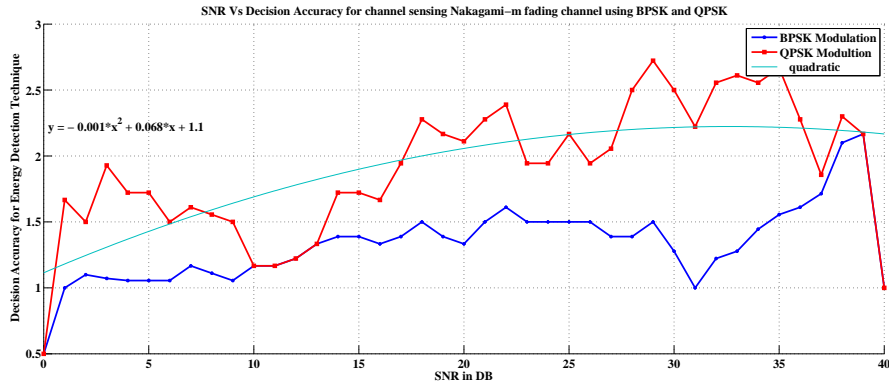

Fig. 9. Decision accuracy of Energy Detection technique over Nakagami-m fading channel using BPSK and QPSK modulation

11. Decision accuracy over the whole SNR range is almost constant for both the channels and modulation techniques . Morever Nakagami-m fading channel offer better and equivalent decision accuracy than AWGN channel for both BPSK and QPSK channel.It can be observed in figure 12.

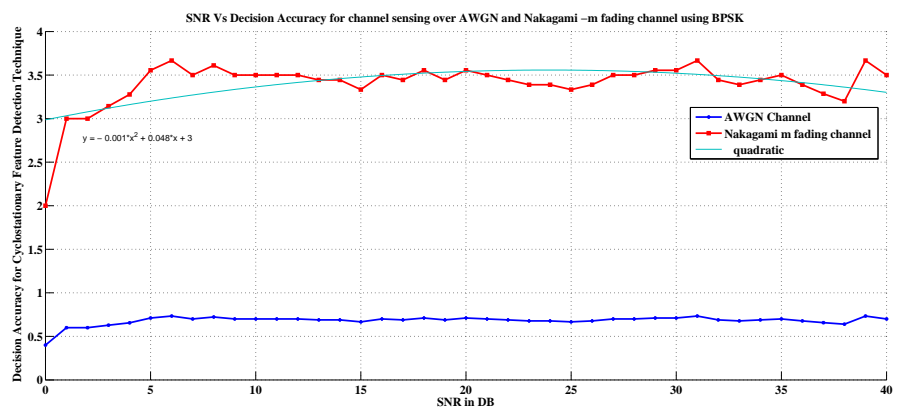

Fig. 10. Decision accuracy of Cyclostationary Feature Detection technique over AWGN and Nakagami-m fading channel using BPSK modulation

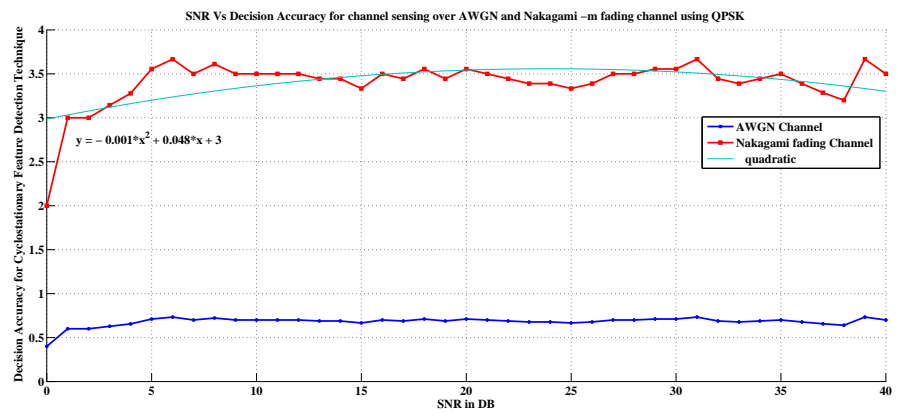

Fig. 11. Decision accuracy of Cyclostationary Feature Detection technique over AWGN and Nakagami-m fading channel using QPSK modulation

\section{CONCLUSION}

Cognitive Radio (CR) has emerged as a leading and promising technology of wireless communication. Exponential growth in wireless communication applications with spectrum scarcity demands dynamic spectrum allocation. Analysis of discussed work gives great results. We can concluded from all the results as Cyclostationary feature detection offers best performance than Matched filtering and energy detection

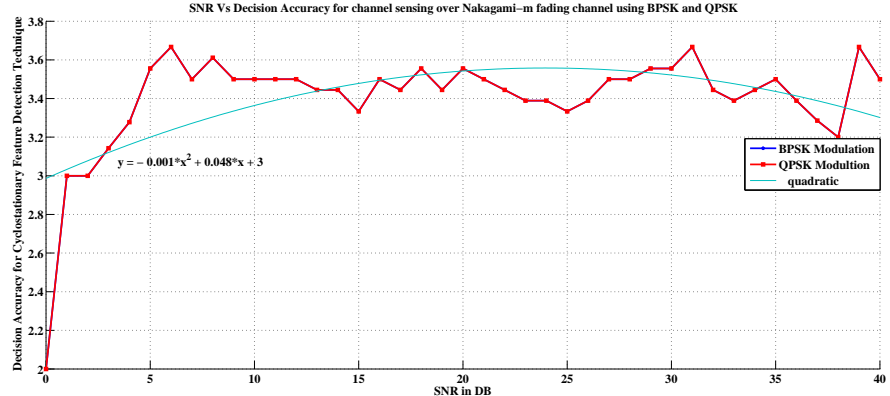

Fig. 12. Decision accuracy of Cyclostationary Feature Detection technique over Nakagami-m fading channel using BPSK and QPSK modulation

technique over Nakagami-m fading channel for both the BPSK and QPSK modulation tecniques. However these both the techniques performs good over AWGN channel for QPSK modulation.

\section{REFERENCES}

[1] Md. Shahnawaz Shaikh, Kamlesh Gupta, "A Review of Spectrum Sensing Techniques for Cognitive Radio",International Journal of Computer Applications (0975 8887),Volume 94 - No. 8, May 2014.

[2] Md. Shahnawaz Shaikh, Kamlesh Gupta "Analysis of Cognitive Radio Spectrum Sensing Techniques", International Journal of Computer Applications (0975 8887), Volume 102 - No. 12, September 2014.

[3] Tevfik Yucek and Huseyin Arslan, A Survey of Spectrum Sensing Algorithms for Cognitive Radio Applications. IEEE COMMUNICATIONS SURVEYS AND TUTORIALS, VOL. 11, NO. 1, FIRST QUARTER 2009.

[4] Mahmood A. Abdulsattar and Zahir A. Hussein, ENERGY DETECTION TECHNIQUE FOR SPECTRUM SENSING IN COGNITIVE RADIO: A SURVEY,International Journal of Computer Networks and Communications (IJCNC) Vol.4, No.5, September 2012.

[5] Simon Haykin, Life Fellow IEEE, David J. Thomson, Fellow IEEE, and Jeffrey H. Reed, Fellow IEEE,Spectrum Sensing for Cognitive Radio, Proceedings of the IEEE. Vol. 0018-9219/ 2009 IEEE 97, No. 5, May 2009.

[6] Ying-Chang Liang, Senior Member, IEEE, Yonghong Zeng, Senior Member, IEEE, Edward C.Y. Peh, and Anh Tuan Hoang, Member, IEEE, Sensing-Throughput Tradeoff for Cognitive Radio Networks,IEEE TRANSACTIONS ON WIRELESS COMMUNICATIONS, VOL. 7, NO. 4, APRIL 2008.

[7] Zhi Quan, Student Member, IEEE, Shuguang Cui, Member, IEEE, and Ali H. Sayed, Fellow, IEEE, Optimal Linear Cooperation for Spectrum Sensing in Cognitive Radio Networks, IEEE JOURNAL OF SELECTED TOPICS IN SIGNAL PROCESSING, VOL. 2, NO. 1, FEBRUARY 2008

[8] Paul D. Sutton, Member, IEEE, Keith E. Nolan, Member, IEEE, and Linda E. Doyle, Member, IEEE, Cyclostationary Signatures in Practical Cognitive Radio Applications, IEEE JOURNAL ON SELECTED AREAS IN COMMUNICATIONS, VOL. 26, NO. 1, JANUARY 2008.

[9] Dong-Chan Oh and Yong-Hwan Lee. Energy Detection Based Spectrum Sensing for Sensing Error Minimization in Cognitive Radio Networks, International Journal of Communication Networks and Information Security (IJCNIS) Vol. 1, No. 1, April 2009.

[10] Md. Shamim Hossain, Md. Ibrahim Abdullah, Mohammad Alamgir Hossain, Energy Detection Performance of SpectrumSensing in Cognitive Radio,I.J. Information Technology and Computer Science, 2012, 11, 1117, DOI: $10.5815 /$ ijitcs.2012.11.02

[11] Saqib Saleem1 and Khurram Shahzad, Performance Evaluation of Energy Detection Based Spectrum Sensing Technique for Wireless Channel,INTERNATIONAL JOURNAL OF MULTIDISCIPLINARY SCIENCES AND ENGINEERING, VOL. 3, NO. 5, MAY 2012

[12] S.Shobana, R.Saravanan, R.Muthaiah, Matched Filter Based Spectrum Sensing on Cognitive Radio for OFDM WLANs, International Journal of Engineering and Technology (IJET) ISSN :0975-4024 Vol 5 No 1 Feb-Mar 2013 
[13] M. Ranjeeth, S. Anuradha, Performance of Nakagami Fading Channel over Energy Detection Based Spectrum Sensing, International Journal of Electronics and Communication Engineering, Vol:8, No:10, 2014

[14] Deepti Kakkar1, Arun Khosla1, Moin Uddin2, Efficient Performance Analysis of Spectrum Sensing for Cascaded Multihop Network over Nagakami-m Fading Channels, Scientific Research, Engineering, 2014, $6,71-77$
Creative Commons Attribution License 4.0 (Attribution 4.0 International, CC BY 4.0)

This article is published under the terms of the Creative Commons Attribution License 4.0

https://creativecommons.org/licenses/by/4.0/deed.en_US 